\title{
Design, Integration and Characterization of a Tracking Patch: Application to Elderly Monitoring
}

\author{
Bouchta Hajjine, Christophe Escriba, Daniel Medale, Jean-Yves Fourniols \\ LAAS-CNRS, Université de Toulouse, CNRS, INSA, Toulouse, France \\ Email: bhajjine@laas.fr, cescriba@laas.fr, medale@laas.fr, fourniols@laas.fr
}

How to cite this paper: Hajjine, B., Escriba, C., Medale, D. and Fourniols, J.-Y. (2016) Design, Integration and Characterization of a Tracking Patch: Application to Elderly Monitoring. E-Health Telecommunication Systems and Networks, 5, 57-74. http://dx.doi.org/10.4236/etsn.2016.53006

Received: July 15, 2016

Accepted: September 27, 2016

Published: September 30, 2016

Copyright $\odot 2016$ by authors and Scientific Research Publishing Inc.

This work is licensed under the Creative Commons Attribution-NonCommercial International License (CC BY-NC 4.0).

http://creativecommons.org/licenses/by-nc/4.0/

\begin{abstract}
According to the latest studies, the French population witnesses a high level of elderly. It follows from this phenomenon health troubles, fragility, and for some people suffering from cognitive problems, a daily need of monitoring and tracking in the fugues cases. It is in this context that comes our research program SACHA (Search and Computerize Human Acts). Our ambition is to develop an electronic patch able to trigger alarms, detect falls and provide geolocation service. Our studies were focused on the conception and the integration of different antennas and functionalities of this system in aim to ensure a good compromise "integration/performances". Several prototypes have been tested and validated in a nursing home.
\end{abstract}

\section{Keywords}

Monitoring, Tracking, Patch, Integration, Fugue, Falls, Alarm

\section{Introduction}

The aging of the French population has become increasingly felt with the arrival to the retirement age of the "baby-boom" generation that began to reverse the age pyramid and place the country in front of a population that requires more and more specific assistance and permanent monitoring [1]. To this phenomenon, we can also add the improvement of the life esperance due to the development and the progress that witnessed the scientific and technological sectors. Who says aging, says also the appearance of health troubles, fragility, dependence... This problematic requires finding new ways that can alleviate the high pressure on medico-social institutes and encourage home

${ }^{1}$ Baby-boom generation: period characterized by a high level of birth that began after the second world war and lasted to the middle of 70 s. 
staying.

HHM (Human Health Monitoring) techniques appear to be an alternative solution to manage aging consequences by putting the new technologies in the service of elderly. Different devices were developed for aging problems like falls detectors, tracking devices, health problems predictors... The HHM systems can take different forms such us smart homes based on the use of sensor networks [2], wearable devices including watches [3], insoles [4], smart textiles [5]... Others types of HHM devices are designed to be glued directly on the skin (electronic patch) [6] or even injected and implanted in the human body [7]. Our laboratory LAAS-CNRS has a wide experience of over two decades in the HHM field. Several projects (PROSAFE [8], HOMECARE, FOOT-TEST, RESPECT [9]) have been conducted on the elderly and dependent persons monitoring including smart homes and wearable systems. These projects covered the analytical aspect by developing algorithms for health and human activities monitoring and the integration aspect by the design of different HHM electronic devices. The second section of this article presents the functionalities of the tracking patch, the antennas design and test are resumed in the third one, the fourth part describes the wireless charging system and the cleanroom process to develop miniaturized printed coils, and finally we present the energetic aspect and the battery management.

\section{Tracking Patch}

Our ambition is to develop a miniature patch for the monitoring of elderly suffering from cognitive problems [10]. The patch is able to trigger alarms in the case of anomalies such as falls or exceeding a safety zone to activate geolocation and tracking. As presented in the Figure 1, the monitored person is either in his house or in a nursing center. The safe zone is defined by a "keep alive" link ( $868 \mathrm{MHz})$ between the patch and an $\mathrm{RF}$ beacon implemented in the place of residence. When the person using the patch exceeds the living zone, the RF link "keep alive" is no longer ensured and the patch detects a fugue case, triggers an alarm and activates the tracking functionality. In this case,

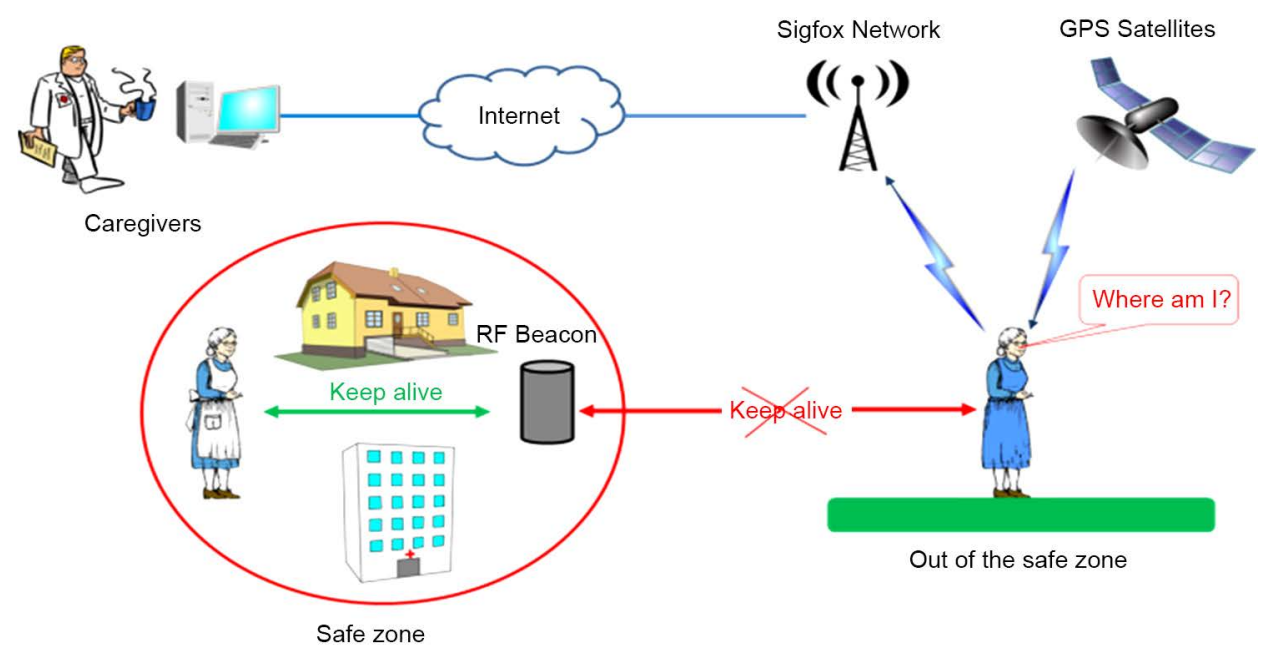

Figure 1. Tracking patch functionalities. 
the GPS coordinates are transmitted by RF communication with Sigfox network based on the ISM $868 \mathrm{MHz}$ band [11] and by this way the caregivers can track and help the concerned person. Falls detection is based on the use of a 3D accelerometer that ensures movement measuring; this functionality will not be presented in this paper.

The tracking patch is developed to be placed on the skin between the shoulder blades (Figure 2). This specific location minimizes tearing and forgetting problems for people with advanced cognitive troubles. To avoid biocompatibility and allergic problems, our system is encapsulated with two medical dressings. As presented in the Figure 2, the tracking patch was miniaturized and integrated in a small packaging of $5.8 \times 2.6 \mathrm{~cm}^{2}$ in aim to avoid any discomfort problems during the use of this device.

\section{RF Links of the Electronic Patch}

\subsection{Design of an ISM $868 \mathrm{MHz}$ and GPS Antennas}

The tracking patch includes two antennas: The first one is used for the communication with the RF beacon and Sigfox network with an operational frequency of $868 \mathrm{MHz}$ and the second antenna that works at a frequency of $1575.42 \mathrm{MHz}$ is used for GPS geolocation. In aim to reduce the cost and bulkiness of our device, we opted for the design of printed antennas [12]. The PIFA (Printed Inverted F Antenna) type was chosen due to its different properties. It is a quarter wave antenna that takes the form of an inverted "F". The radiating arm is shorted to the ground plane to create an inductive effect and in our case the antenna is fed using a coplanar wave guide (Figure 3). The ground plane dimension has an important role to determine the efficiency of the antenna. For our design, it was chosen to ensure both high integration with acceptable performances.

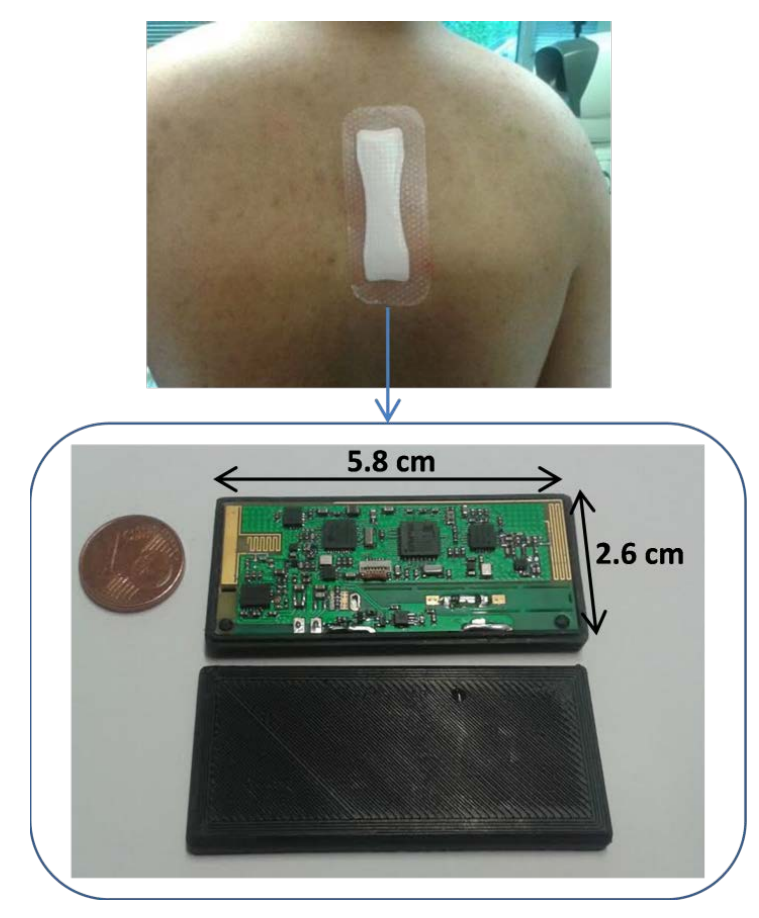

Figure 2. Tracking patch. 


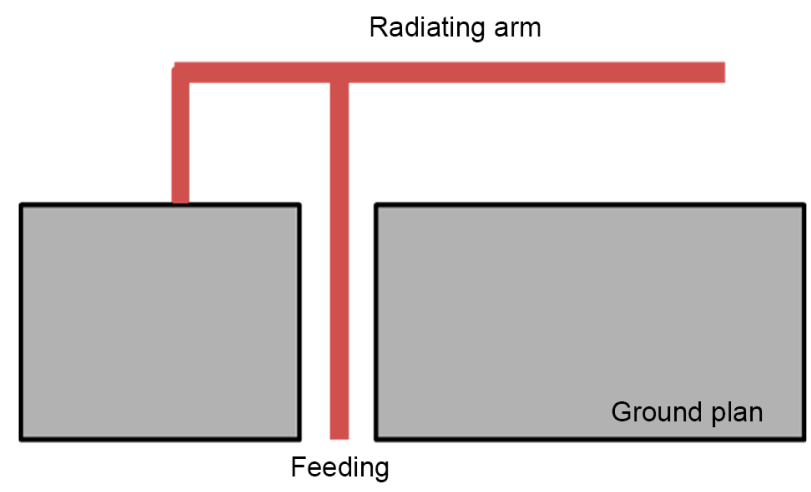

Figure 3. Printed Inverted F Antenna (PIFA).

For a PIFA, The length of the radiating arm must be equal to the quarter of the wave length $(\lambda / 4)$. This criterion is not compatible with the miniaturization constraints of the patch for the ISM and L1 GPS bands: $\lambda / 4=8.64 \mathrm{~cm} @ 868 \mathrm{MHz}$ and $\lambda / 4=4.76 \mathrm{~cm} @$ 1575.42 MHz. To resolve this technological challenge, a miniaturization technique based on the use of meanders was adopted (Figure 4). This method consists of folding the radiating arm many times to minimize the area occupied by the antenna. Modeling works using RF software ADS (Advanced Design System) [13] and HFSS (High Frequency Structural Simulator) [14] were conducted to design the antennas and study the influence of different physical parameters: layers stacking, type of materials, dimensions, meanders form, distance between meanders, coupling phenomena... The ISM and GPS antennas designs using HFSS software are presented in Figure 4.

The proposed antennas were carried out on FR4 substrate and characterized using a vector network analyzer (VNA) E5061B (Agilent Technologies). Figure 5 shows a comparison between different simulated and measured reflection coefficients that present good agreement. This parameter is expressed in decibels $(\mathrm{dB})$ and describes the reflected power from the antenna. A reflection coefficient that tends to $0 \mathrm{~dB}$ means that the energy supplied to the antenna is totally reflected and there is no radiation in this case. This coefficient should be as small as possible and generally we accept a value lower than $-10 \mathrm{~dB}$.

The different values of reflection coefficients and bandwidths are presented in the Table 1. The designed antennas provide quite good performances: for the ISM band, the $868 \mathrm{MHz}$ antenna has a reflection coefficient S11 $=-16.4 \mathrm{~dB}$ with a bandwidth of 16.2 $\mathrm{MHz}$ and for the GPS antenna, it has a reflection coefficient S22 $=-19 \mathrm{~dB}$ with a bandwidth of $39 \mathrm{MHz}$.

\subsection{Tests of RF Communication}

In aim to validate the good functioning of the RF communication with Sigfox network, tests in real cases were carried out. The principle was based on a geographical meshing around our laboratory LAAS-CNRS to select reference points. From each point 10 frames were initially sent and in the end we compared the number of received frames by Sigfox stations implemented in Toulouse (France) to estimate the reception quality. 


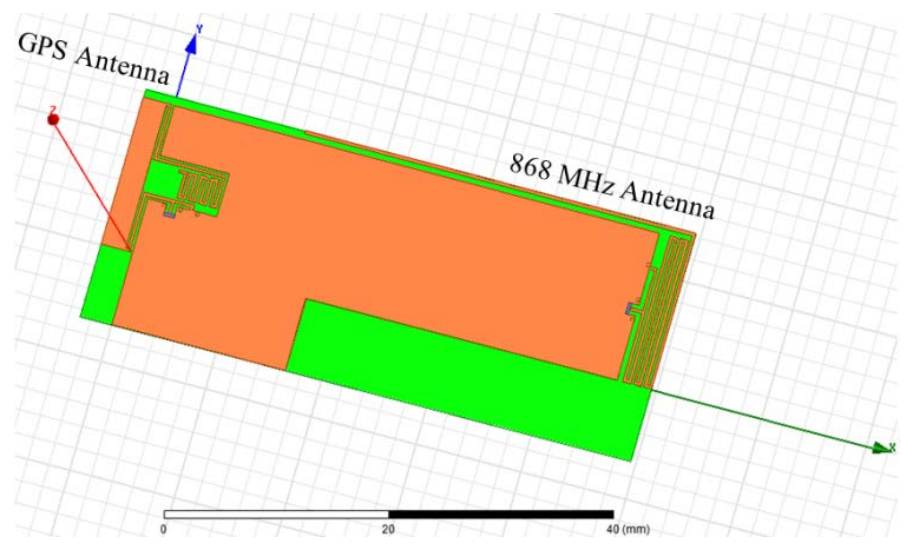

Figure 4. Antennas design and simulation using HFSS software.

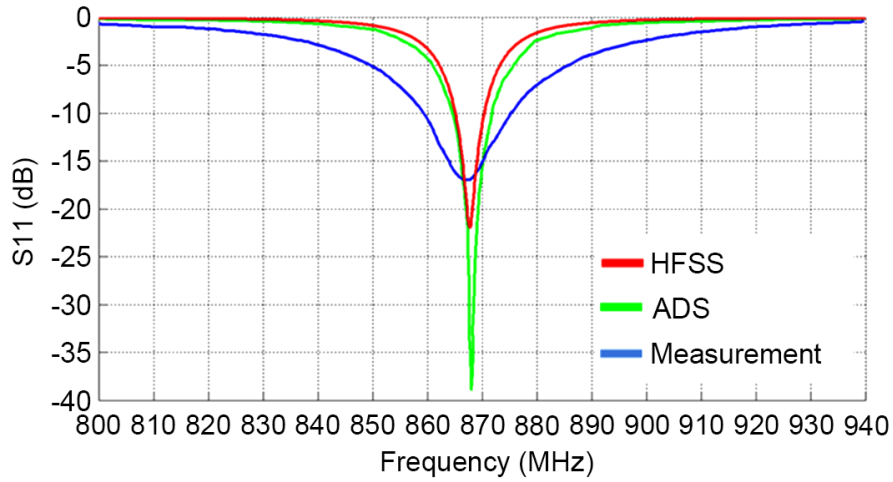

(a)

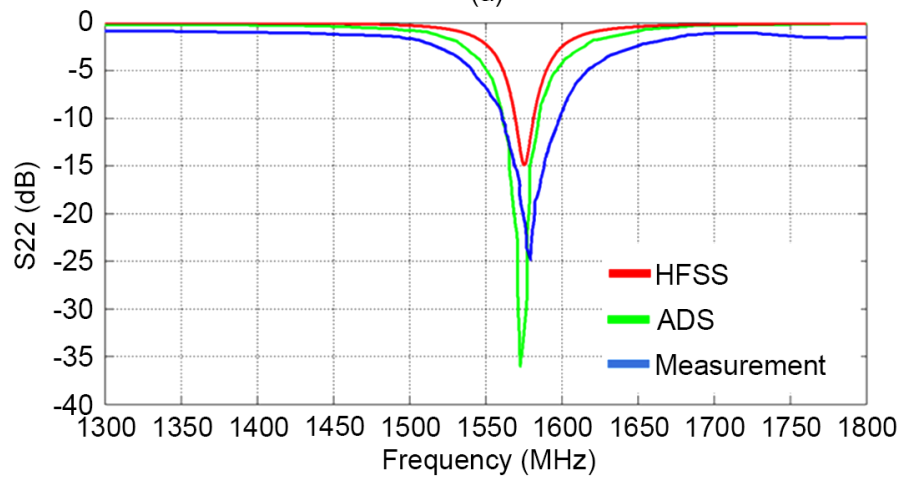

(b)

Figure 5. Comparison of simulated and measured reflection coefficients: S11 for ISM antenna and S22 for GPS antenna.

Table 1. Antennas reflection coefficients and bandwidths.

\begin{tabular}{ccccc}
\hline & \multicolumn{2}{c}{ ISM Antenna } & \multicolumn{2}{c}{ GPS Antenna } \\
\cline { 2 - 5 } & S11 @ $868 \mathrm{MHz}$ & BW & S22 @ $1575.42 \mathrm{MHz}$ & $\mathrm{BW}$ \\
\hline ADS & $-38 \mathrm{~dB}$ & $7.3 \mathrm{MHz}$ & $-36.8 \mathrm{~dB}$ & $25 \mathrm{MHz}$ \\
HFSS & $-21 \mathrm{~dB}$ & $5.3 \mathrm{MHz}$ & $-15 \mathrm{~dB}$ & $12 \mathrm{MHz}$ \\
Measurement & $-16.4 \mathrm{~dB}$ & $16.2 \mathrm{MHz}$ & $-19.8 \mathrm{~dB}$ & $39 \mathrm{MHz}$ \\
\hline
\end{tabular}


Two cases were studied, for the first one the tracking patch was not placed on the skin and for the second test the patch was glued on the skin to take into account the influence of the human body.

The results of the first test are resumed in the Figure 6: when the patch is not placed on the skin, for all the tested points the reception quality is greater than $60 \%$. This means that we receive each time more than 6 frames. In addition, for $80 \%$ of the reference points we receive all the 10 frames initially sent.

Table 2 presents the number of received frames of all reference points in the first test. As the patch is developed to be glued on the skin, these results will be compared with the second test that takes into consideration the presence of the human body.

For the second test, the same principle of geographical meshing was kept and this time the patch was glued on the human body of a volunteer. The reception qualities of the different tested points are presented in Figure 7 and Table 3.

From Figure 7, we can notice that when the patch is placed on the human body, only two points have a reception quality lower than $60 \%$ (points in yellow color). In addition, for $68 \%$ of the tested points we are able to receive all the frames initially sent ( $\mathrm{Ta}$ ble 3). These tests validate the good functioning of the communication with Sigfox network when the patch is glued on the human body.

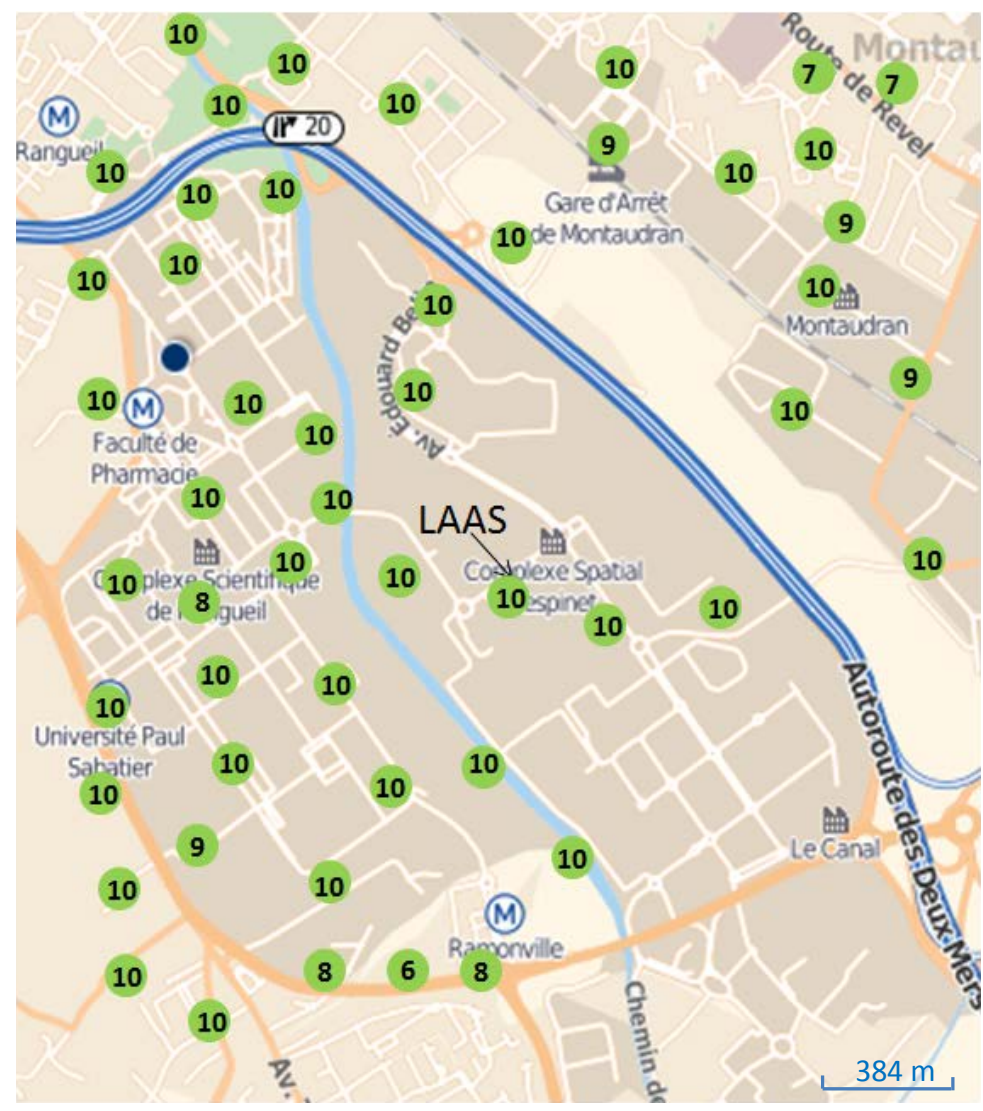

Figure 6. Test of the RF communication with Sigfox network when the patch is not placed on the human body. 
Table 2. Results of the first RF communication test (patch not placed on the skin).

\begin{tabular}{cc}
\hline Points & Reception quality \\
$80.39 \%$ & 10 \\
$7.84 \%$ & 9 \\
$5.88 \%$ & 8 \\
$3.92 \%$ & 7 \\
$1.96 \%$ & 6 \\
$0 \%$ & $\leq 5$ \\
\hline
\end{tabular}

Table 3. Results of the second RF communication test (patch placed on the human body).

\begin{tabular}{cc}
\hline Points & Reception quality \\
$67.92 \%$ & 10 \\
$13.2 \%$ & 9 \\
$0 \%$ & 8 \\
$11.32 \%$ & 7 \\
$3.77 \%$ & 6 \\
$3.77 \%$ & $\leq 5$ \\
\hline
\end{tabular}

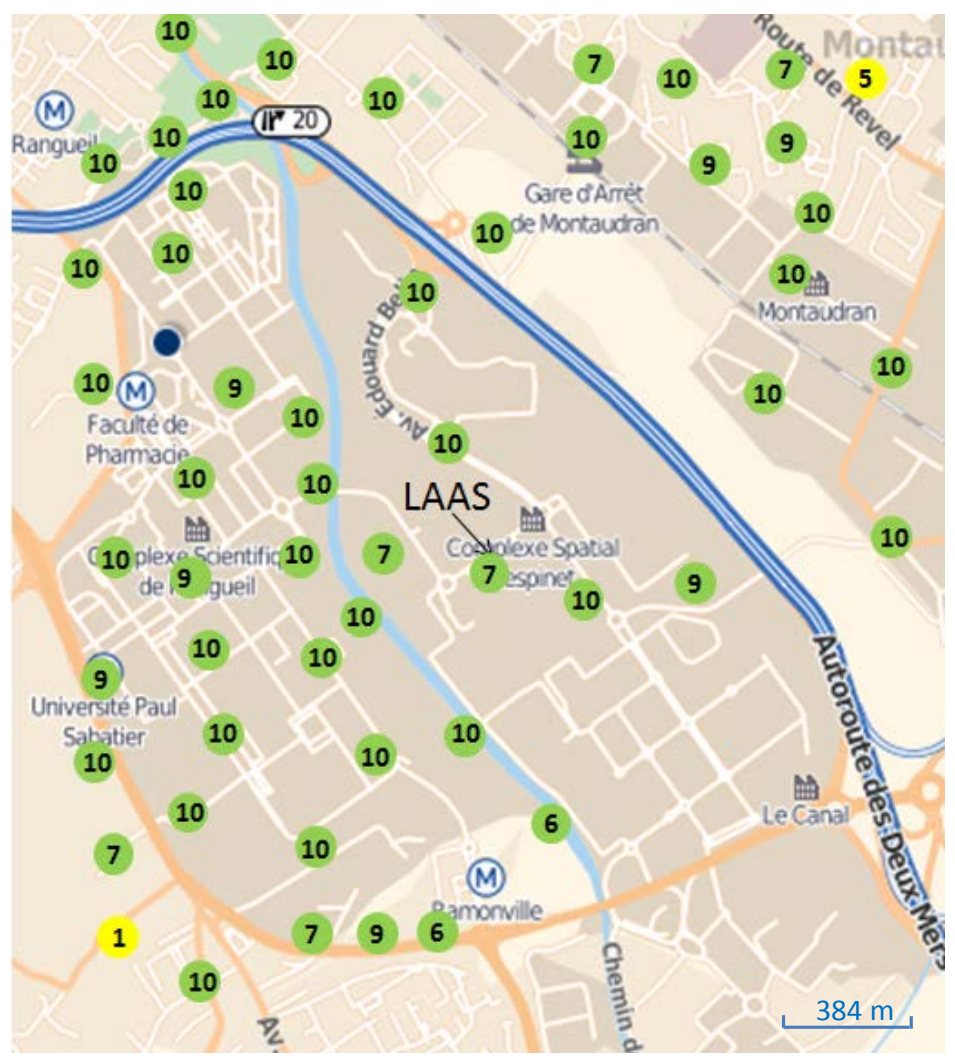

Figure 7. Test of the RF communication with Sigfox network when the patch is placed on the human body. 


\section{Wireless Charging of the Tracking Patch}

\subsection{Wireless Energy Transfer Functionality}

In recent years, wireless chargers appeared increasingly on the market. This solution seems the most suitable for our device. It is simple to use, we just need to put the patch on a charging pad without using any cable which allows avoiding the problem of incompatibility between different chargers. Furthermore, by choosing a suitable packaging the waterproof property can be ensured. A comparative study of different standards and protocols known in this field has allowed us to select the Qi protocol using magnetic induction [15].

As explained in Figure 8, the energy transfer is ensured by magnetic coupling between a receiver (encapsulated patch) and an emitter (charging pad). The Qi protocol adds a unidirectional communication (messages from the receiver to the transmitter) to control the different charging states: full charging of the battery, presence of a Qi compatible receiver, presence of metallic objects...

The wireless charging functionality needs the use of coils to recuperate energy by magnetic induction. Given the patch integration requirements, the commercialized coils cannot be used for our system due to their bulky forms. An example is presented in Figure 9, this coil has a dimension of $3.25 \times 4.85 \mathrm{~cm}^{2}$ and cannot be integrated in the patch (Figure 9).

To overcome this issue, our efforts were focused on the design of a thin printed coil to meet the integration requirements by placing it under the battery of the patch. A ferrite layer was also added to ameliorate the energy transfer performances. This layer acts
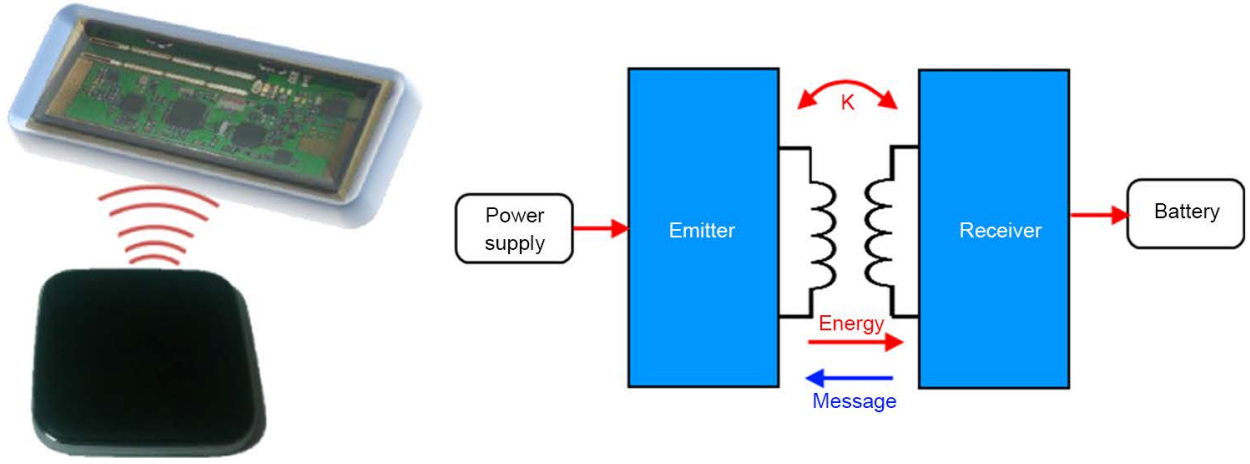

Figure 8. Wireless energy transfer by magnetic induction.

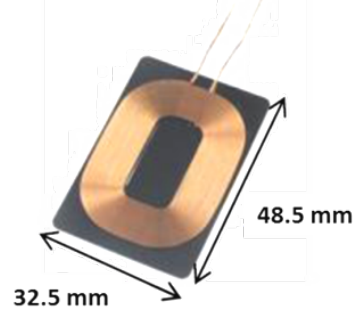

Figure 9. Example of commercialized coil for wireless charging (WR483250-15M2-G). 
as a magnetic core to channel the magnetic field and avoid the influence of the battery on the coil performances. The final stacking of the different layers constituting the tracking patch is presented in the Figure 10: the PCB of the patch, the battery, the coil and the ferrite layer are encapsulated with a plastic packaging and a medical dressing is also used to avoid any allergic or discomfort problems.

\subsection{Design of a Printed Coil for Wireless Power Transfer}

The proposed coil is composed of two layers of copper with a thickness of $17 \mu \mathrm{m}$ separated by a $50 \mu \mathrm{m}$ of polyimide substrate. The coil dimensions were chosen to meet the battery area $\left(40 \times 18 \mathrm{~mm}^{2}\right)$. The $\mathrm{Cu}$ tracks have a width of $520 \mu \mathrm{m}$ and they are separated by a spacing of $80 \mu \mathrm{m}$. Figure 11 presents a 3D view of the proposed printed coil obtained using ADS software. The two layers of copper are connected in series using vias.

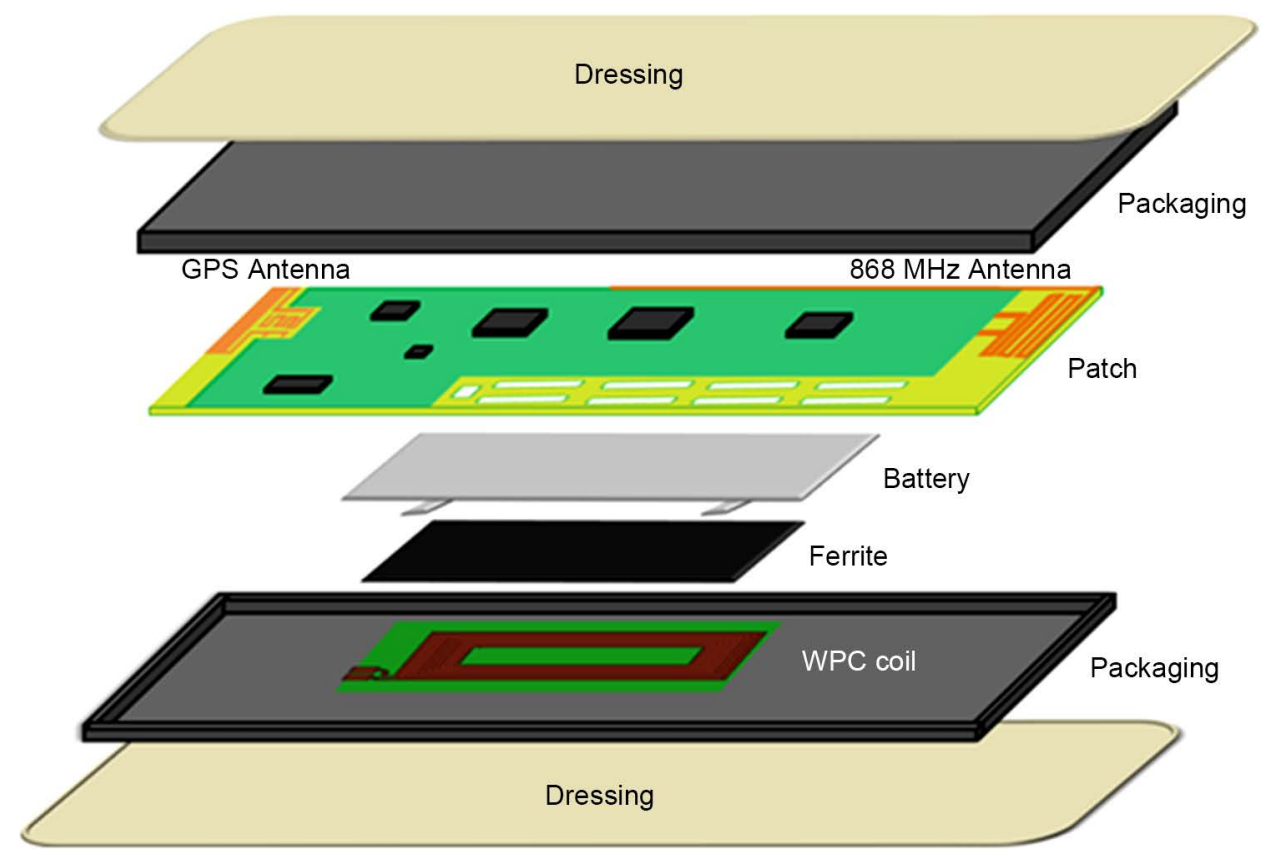

Figure 10. Stacking of the patch layers.

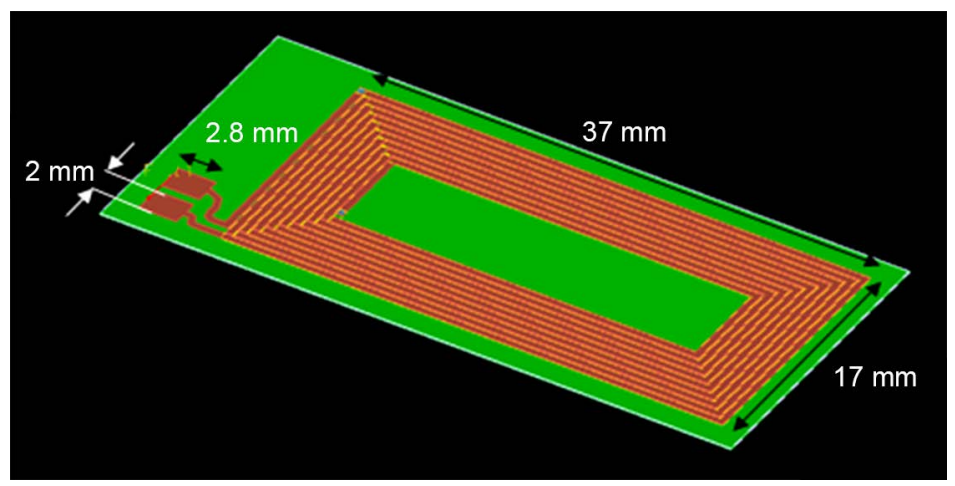

Figure 11. Design of a printed coil for the wireless energy transfer. 
Design, simulations and validation of the performances of the printed coil were conducted using ADS and HFSS software. The variations of inductance, resistance, and quality factor of the proposed model are plotted in the Figure 12 as predicted by the both softwares.

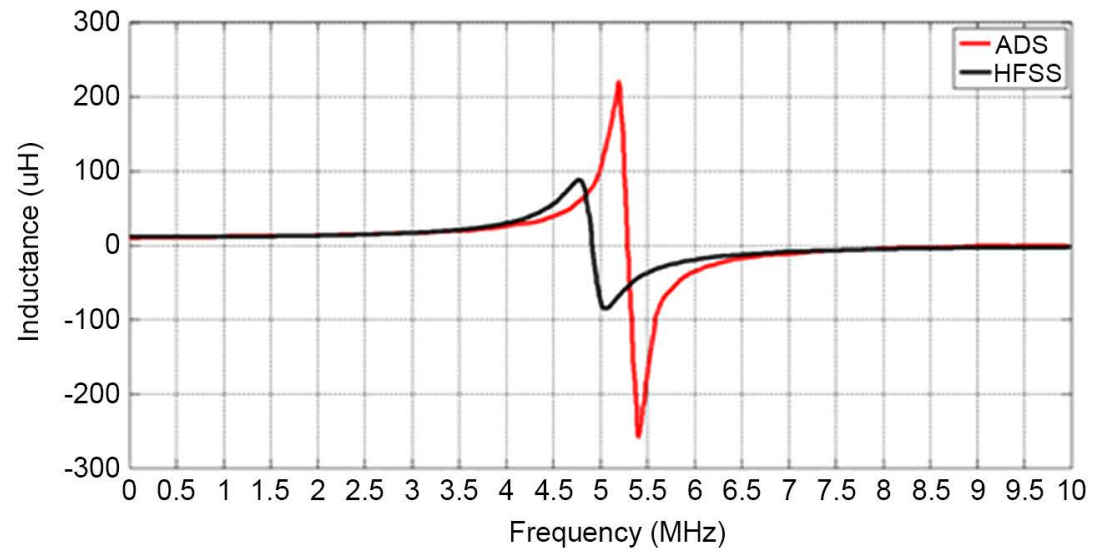

(a)

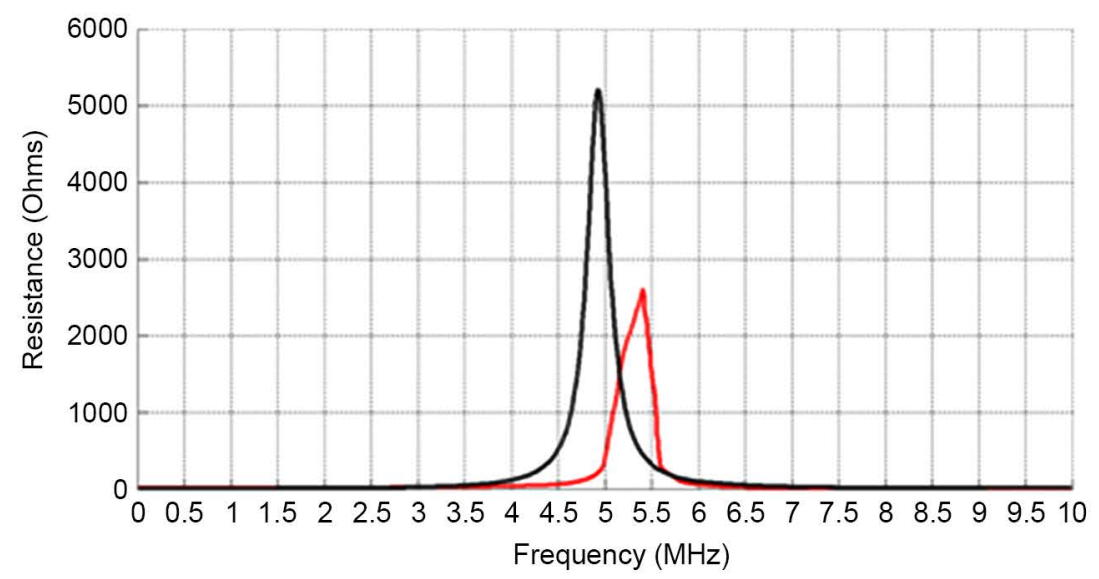

(b)

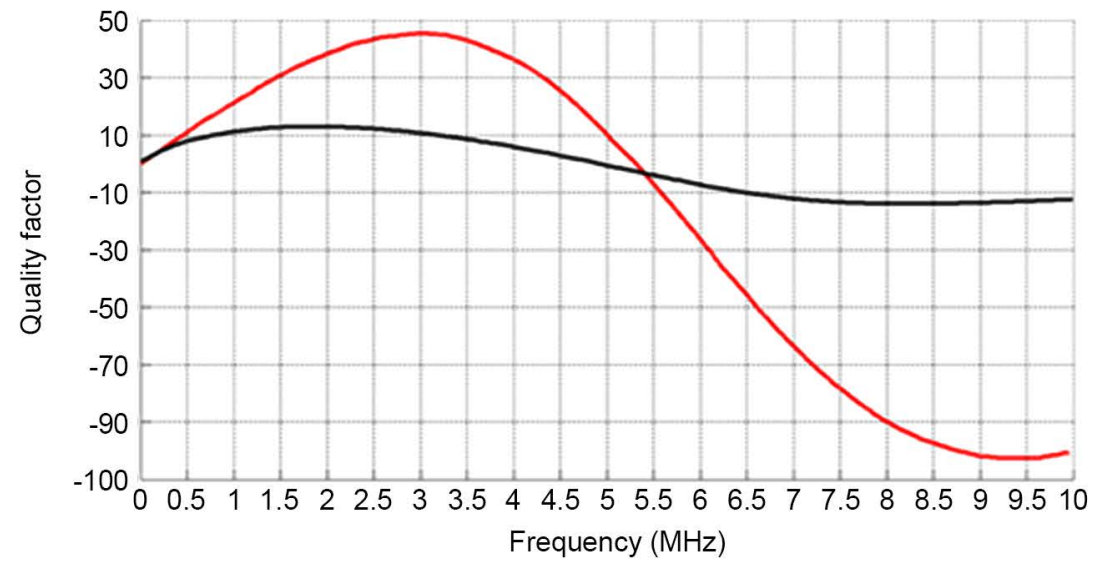

(c)

Figure 12. Simulated performances of the proposed printed coil: (a) inductance $(\mu \mathrm{H})$, (b) resistance $(\Omega)$, and (c) quality factor. 
From Figure 12, we can notice that simulations predict a self-resonance frequency of 5.3 $\mathrm{MHz}$ obtained with ADS and 4.9 MHz computed using HFSS. These values are compatible with the frequency operating range used by the protocol Qi $(110-205 \mathrm{kHz})$ : they are much greater than the maximum value $(205 \mathrm{kHz})$ and this property ensures constant values of the electrical performances $(\mathrm{L}, \mathrm{R}$, and $\mathrm{Q})$ in this range.

For the charging frequency interval, the model simulated with ADS presents an inductance of $10.74 \mu \mathrm{H}$, a resistance of $3 \Omega$, and a quality factor of $3.37 @ 150 \mathrm{kHz}$. Concerning the results obtained with HFSS software, the coil has an inductance of 10.71 $\mu \mathrm{H}$, a resistance of $3.25 \Omega$, and a quality factor of $3.1 @ 150 \mathrm{kHz}$.

\subsection{Cleanroom Process for the Fabrication of the Printed Coil}

The printed coils were carried out in the cleanroom of our laboratory LAAS-CNRS. A polyimide photoresist HD-4110 [16] was used to form different cover layers and the substrate separating the two layers of $\mathrm{Cu}$ tracks. By using this photoresist the different openings (contact pads and vias) were obtained directly by photolithography. In addition, another non photoresist of polyimide PI2611 [17] was also used to form a sacrificial layer between the Si wafer and the different deposited layers by spin coating technique. Unlike our last process described in your article [18], this time the vias were filled with copper to ensure a better connection between the two copper layers. The copper tracks and vias were made by electroplating via a mold of BPN photoresist to obtain directly the desired forms. This operation was preceded by an evaporation phase of a seed layer $\mathrm{Ti} / \mathrm{Cu}(100 \mathrm{~nm} / 100 \mathrm{~nm})$ needed for the electrolytic deposition of copper. The different technological steps of this process are presented in Figure 13.

A prototype of the printed coil is presented in Figure 14(a). The dimensions of the connecting vias and copper tracks are illustrated in Figures 14(b)-(d). Due to the inclined profile of electroplated copper, two characteristic values are given each time: diameter/distance in the top and the bottom of the vias, tracks, and the inter-tracks spacing.

The printed coil was characterized using an impedance analyzer Agilent 4294A (Figure 15) to study the variations of the electrical performances (inductance, resistance, and quality factor) in function of frequency in aim to validate the simulated values computed using RF softwares.

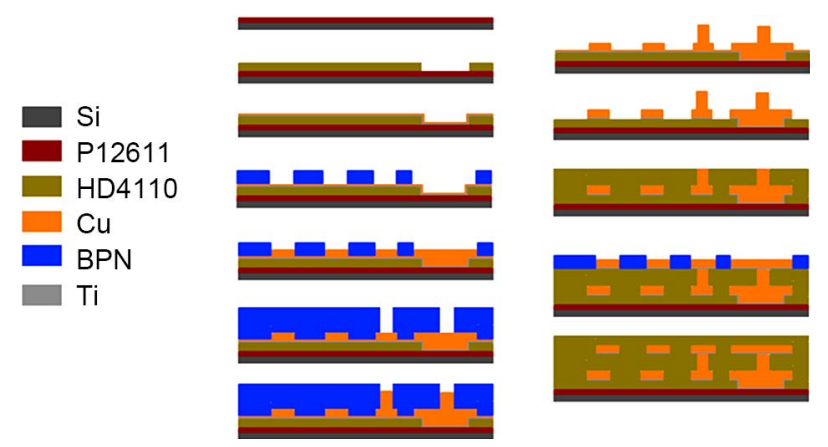

Figure 13. Technological steps of the cleanroom process. 


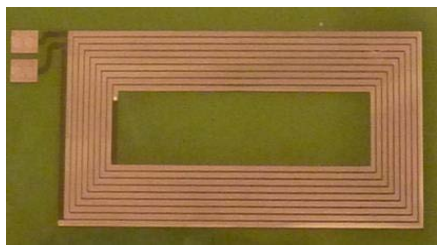

(a)

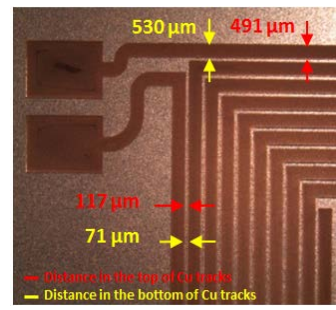

(c)
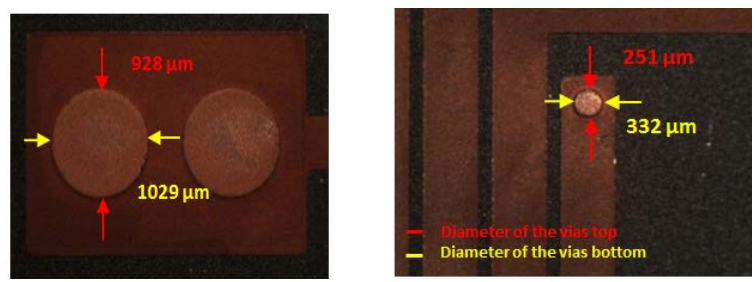

(b)

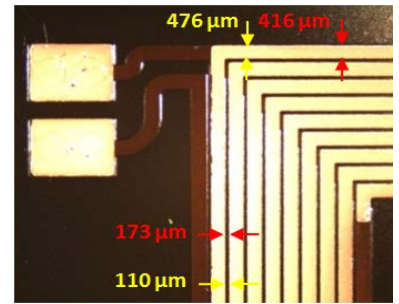

(d)

Figure 14. Printed coil carried out in the cleanroom of LAAS-CNRS. (a) Printed coil for wireless charging; (b) Electroplating of vias; (c) First Cu layer; (d) Second Cu layer.

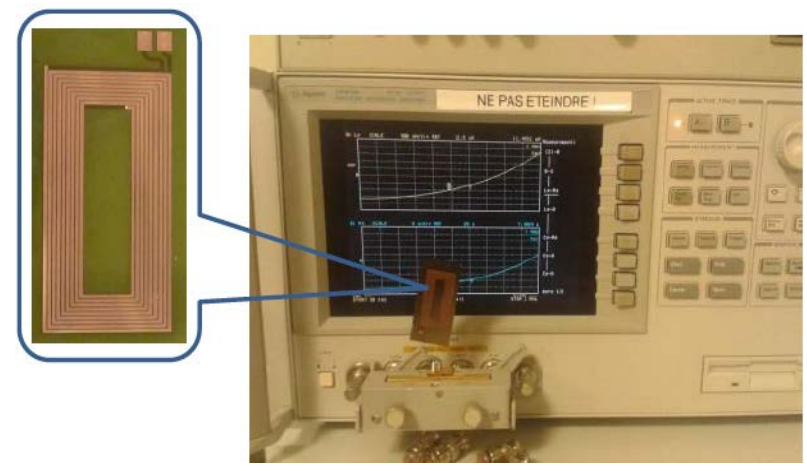

Figure 15. Characterization of the printed coil.

The measured results are plotted in the Figure 16, the resonance frequency is shifted (3.9 $\mathrm{MHz}$ ) compared to the simulation results (5.3 $\mathrm{MHz}$ obtained with ADS and 4.9 $\mathrm{MHz}$ computed with HFSS). This frequency shift does not disturb the coil performances because it is still far from the frequency range used by the Qi wireless charger $(110 \mathrm{kHz}-205 \mathrm{kHz})$. For this range, the measurement results meet the simulated values: $\mathrm{L}=10.8 \mu \mathrm{H}, \mathrm{R}=3 \Omega$, and $\mathrm{Q}=3.39$ @ $150 \mathrm{kHz}$.

After the validation of the electrical performances of the printed coils, the wireless charging system was integrated in the tracking patch. Several charging pads compatible with the Qi standard were used to validate the interoperability property and the charging current allure. In the Figure 17, the patch is encapsulated using a plastic packaging and is being charged wirelessly using the magnetic induction created by the charging pad. For this test, the charging current value is $10 \mathrm{~mA}$.

A thermal study of the patch is necessary to investigate and quantify temperature changes during the wireless charging (Eddy current created by the magnetic field). This temperature variation must not exceed a maximal value of $45^{\circ} \mathrm{C}$ to avoid the deterioration 


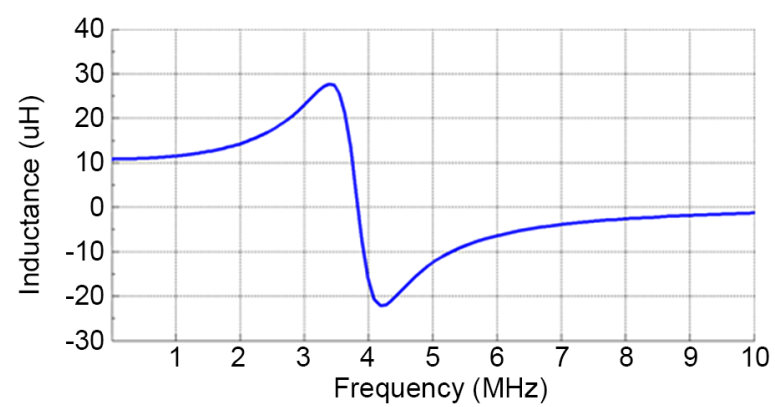

(a)

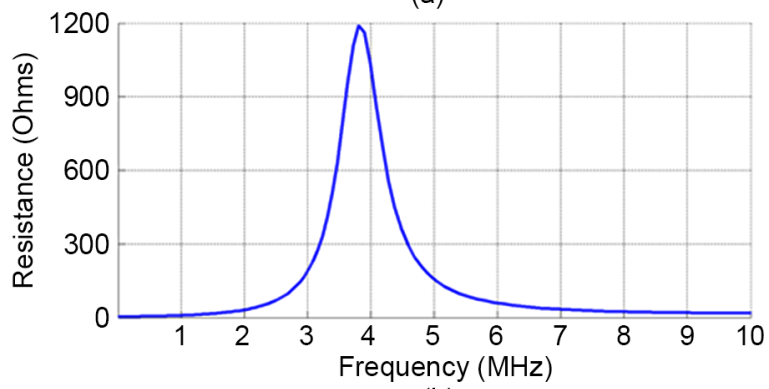

(b)

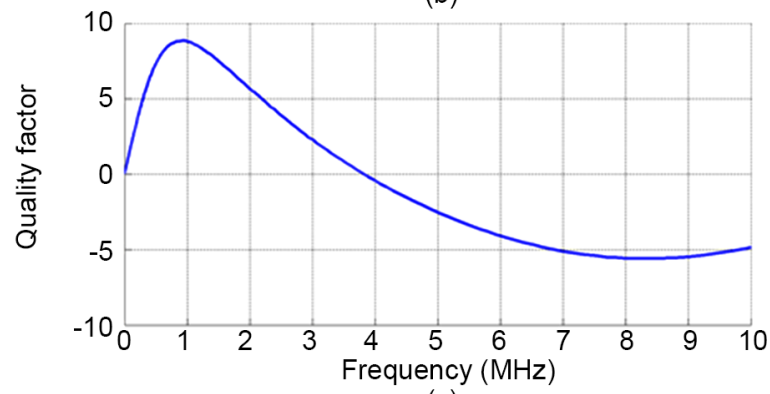

(c)

Figure 16. Measured performances of the printed coil: (a) inductance $(\mu \mathrm{H})$, (b) resistance $(\Omega)$, and (c) quality factor.
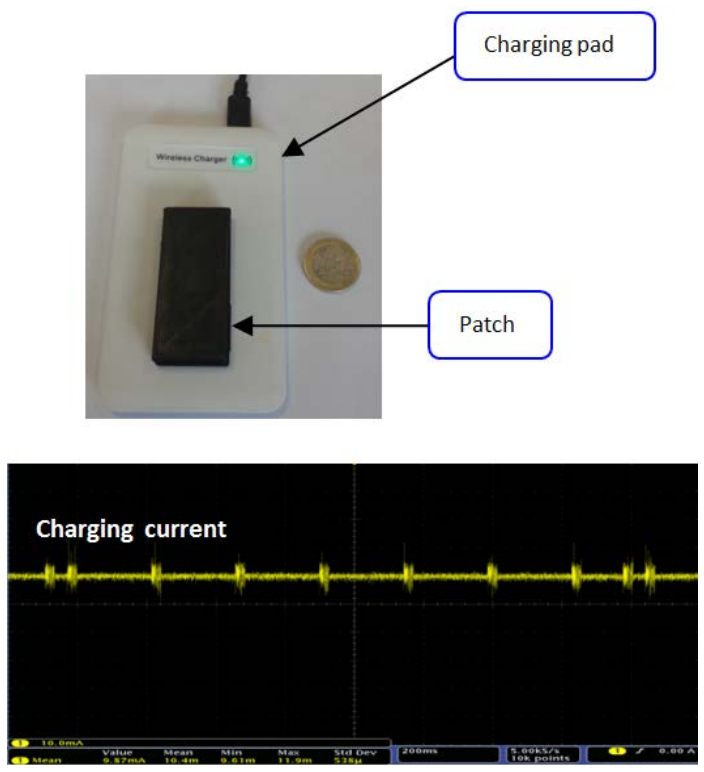

Figure 17. Test of the wireless charging of the patch. 
of the battery performances. To conduct our investigation, a thermal camera "FLIR x6580sc" was used. The patch and the charging pad were placed at a distance of $50 \mathrm{~cm}$ from the camera lens and the sampling frequency was adjusted to obtain 5 images/s (Figure 18).

The Analysis of the test results reveals that the maximum temperature noticed during the wireless charging of the patch does not exceed $32^{\circ} \mathrm{C}$ that is lower than the maximum value for the using of the battery $\left(45^{\circ} \mathrm{C}\right)$. The variation of the maximal temperature of the patch during 1 hour of charging is plotted in the Figure 19. This figure is obtained directly using the above mentioned camera by selecting the area that witnesses the maximum value of temperature in the thermal image treated by the camera software (Figure 18).

This test confirms that the patch is well protected from the temperature elevation that can be caused by the magnetic field (Foucault currents) during the wireless charging.

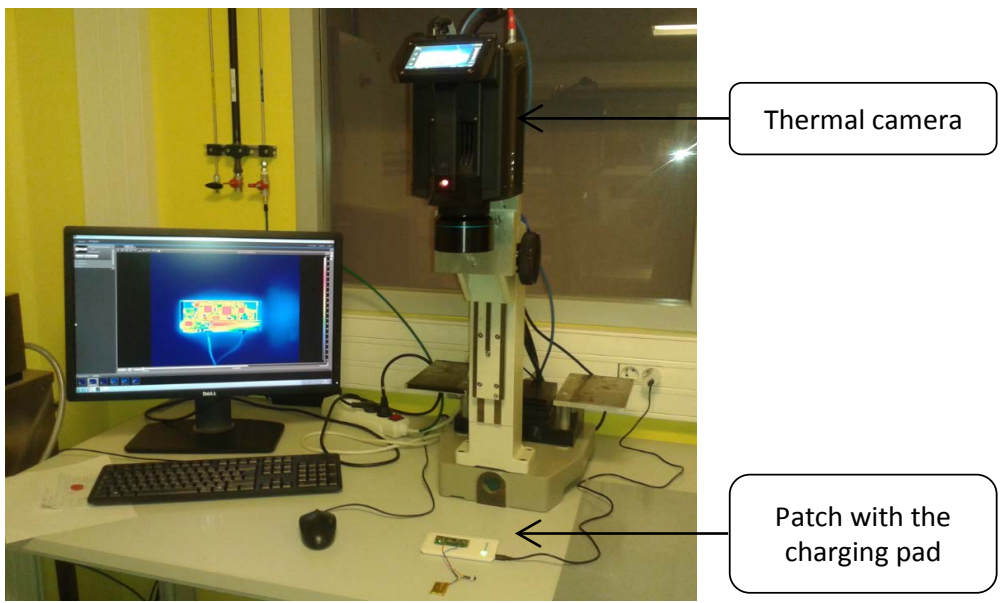

Figure 18. Thermal test of the tracking patch.

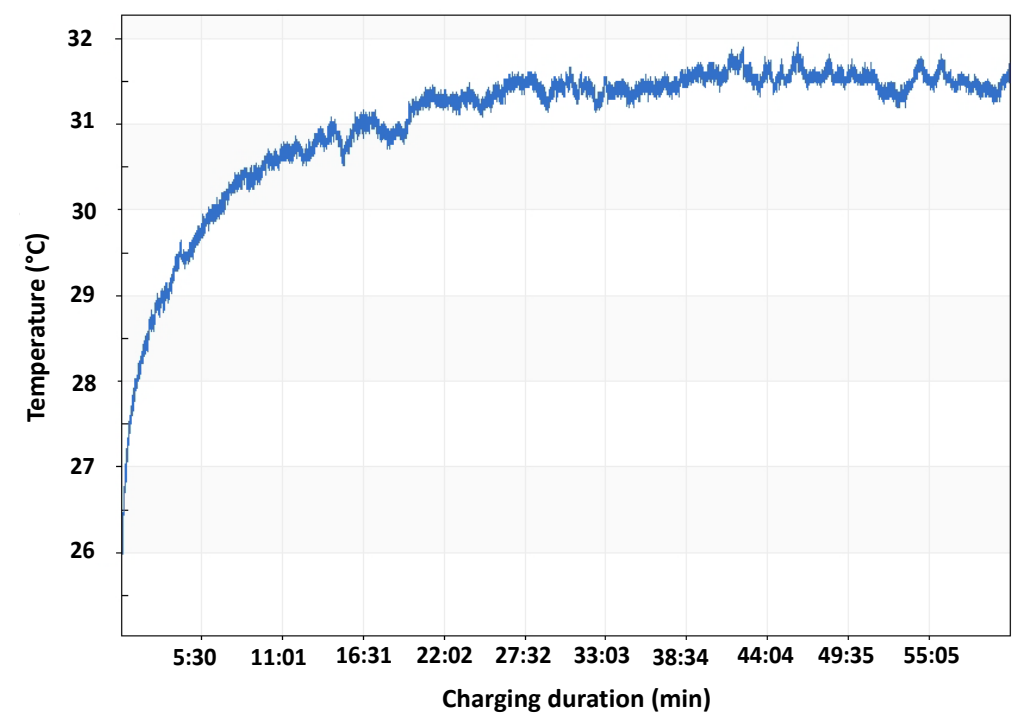

Figure 19. Variation of the patch temperature during the wireless charging. 


\section{Energetic Aspect and Battery Management}

The tracking patch is equipped with a tiny battery ( $1 \mathrm{~mm}$ thick) to ensure a nominal operational functioning of 3 days and a using duration of 13 hours after the GPS activation when a fugue is detected. The charging duration is $4-5$ hours using the wireless energy transfer described in the previous section (charging current $=7.5 \mathrm{~mA}$ ).

Knowing that the patch is intended to be fully encapsulated to ensure waterproof property, a remote system is necessary for the activation and deactivation of the patch (HMI). In our case, the RF solutions are inadequate and require an additional specific design of a new antenna. Using external magnetic field seems more appropriate. The charging system is based on the magnetic induction and we cannot reuse the same principle for the patch command. A Hall effect sensor requires a power supply that causes a supplementary energy consumption for the patch. For these reasons, we opted for the use of a passive switch REED.

The integration of a switch REED in the PCB of the patch is illustrated in the Figure 20. This switch consists of a glass ampoule encapsulating two moving metal strips that are magnetized and move to close the switch in the presence of an external magnetic field. These transitions low-high voltage levels ensured by this switch can be detected by the microcontroller to activate or deactivate the patch.

To avoid excessive discharge of the battery that can affect its performances, the tracking patch is equipped with an undervoltage protection functionality. The protection threshold is $2.8 \mathrm{~V}$, a recommended value by the battery manufacturer and the reactivation threshold is $3.3 \mathrm{~V}$, a fixed value by the characteristic of the chosen protection component. This functionality is tested in the Figure 21: for the first case the patch is deactivated when the battery voltage attains a value of $2.86 \mathrm{~V}$. To reactivate the patch the battery must be charged to exceed a value of $3.34 \mathrm{~V}$.

A new patch deactivation functionality is necessary to avoid energy consumption during the charging phases. This property is based on the use of PMOS transistors controlled via the charging status ensured by the component that manages the wireless energy transfer. This functionality is tested in the Figure 22, we can notice that the feeding power is cut off when the patch is placed on a charging pad.
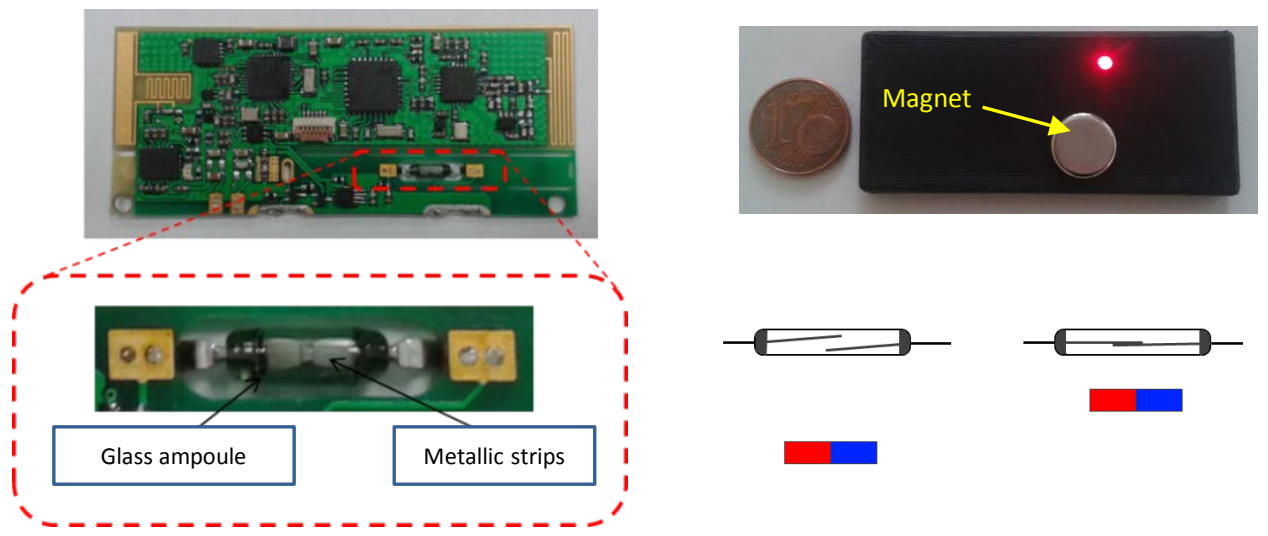

Figure 20. Activation of the tracking patch using an external magnet and a switch REED. 


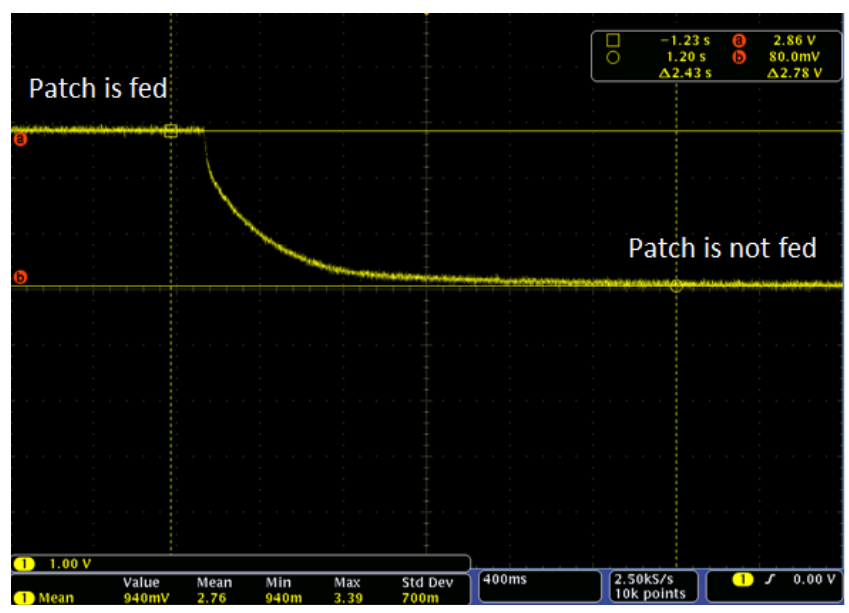

(a)

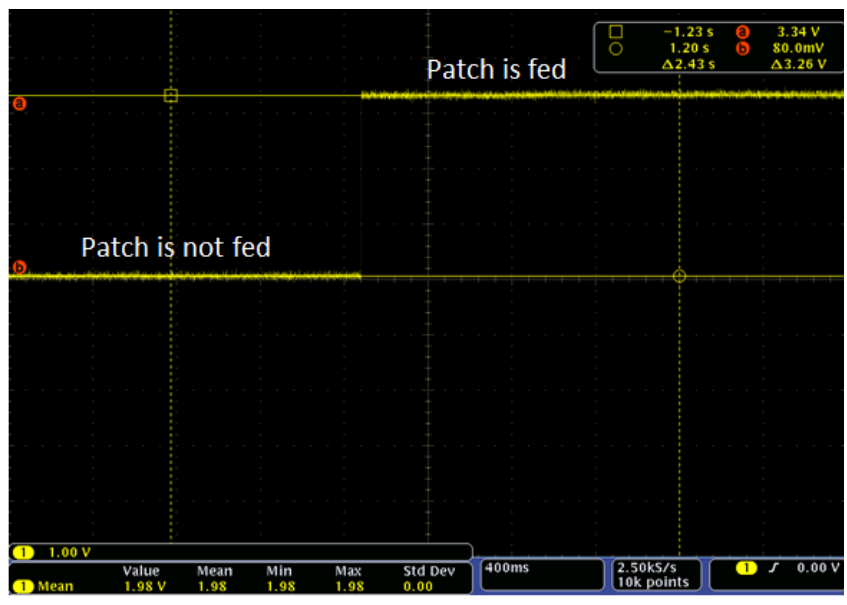

(b)

Figure 21. Undervoltage protection ((a) deactivation @ Vbat=2.86 V and (b) activation @ Vbat = 3.34 V).

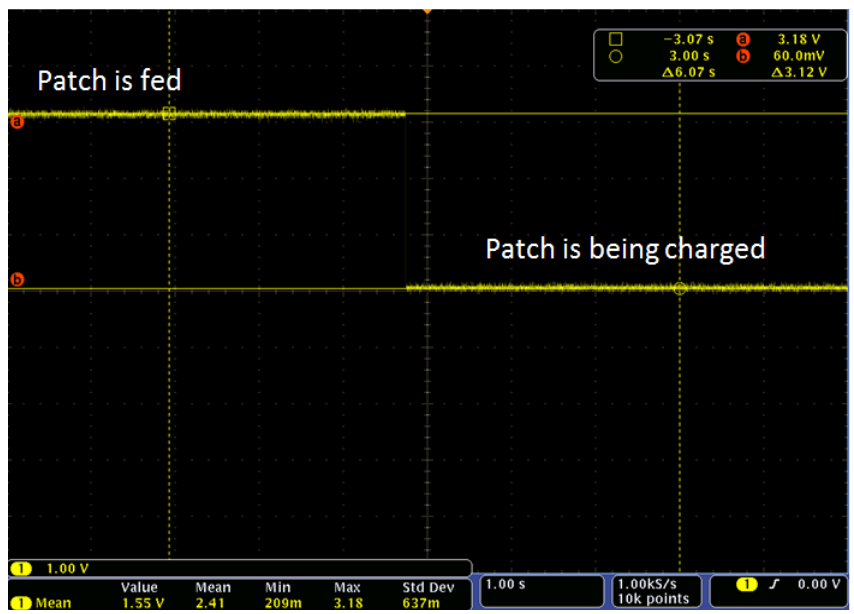

(a)

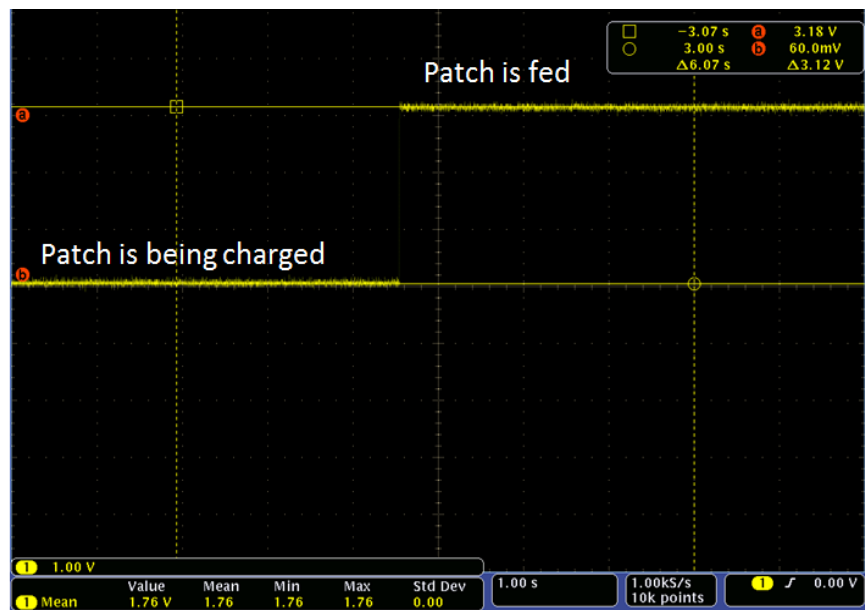

(b)

Figure 22. Feeding voltage of the patch ((a) presence of the wireless charger and (b) Absence of the charging pad).

\section{Conclusion}

This paper describes the design of an electronic tracking patch for the monitoring of elderly suffering from cognitive problems. This device provides two principle functionalities: falls detection and GPS tracking. For this kind of systems, the miniaturization is a very important criterion to ensure users acceptability and avoid any discomfort problems. This constraint is always faced by integration problems: bulky antennas, energetic consumption. The different works discussed in this paper present a complete study (design, simulation, cleanroom process, optimization...) of a tracking system and highlight technological challenges and associated solutions. Our efforts were initially focused on the conception and integration of antennas to ensure data transfer and geolocation. The proposed designs were studied by RF simulation and their performances were validated by measurements and real cases tests. To ensure an easy use and waterproof property, a wireless charging system was integrated. The commercialized coils 
used in wireless energy transfer are bulky and do not meet the integration requirement of the tracking patch. For this reason, we decided to develop a miniaturized printed coil. This coil was studied using RF software and carried out in the cleanroom of LAASCNRS. After the validation of the good functioning of the wireless charging, others complementary functionalities were added to manage the energetic aspect of the patch: remote activation system, undervoltage protection, deactivation of the patch during the battery charging. These works permitted us to develop a miniaturized tracking patch that was validated in real scenarios in a nursing home.

\section{Acknowledgements}

This work is part of SACHA project funded by French government and "Région Midi Pyrénées” in France. The partners of the project are Sigfox, Axible, Telecom Design companies, e-santé and CHIVA hospital.

This work was partly supported by LAAS-CNRS micro and nano technologies platform member of the French RENATECH network.

\section{References}

[1] French Population. http://www.insee.fr/fr/themes/tableau.asp?ref_id=ccc

[2] Chan, M., Campo, E., Estève, D. and Fourniols, J.-Y. (2009) Smart Homes-Current Features and Future Perspectives. Maturitas, 64, 90-97.

http://dx.doi.org/10.1016/j.maturitas.2009.07.014

[3] Vivago. http://www.vivago.fr/SAS/index.php

[4] GPS Smart Sole. http://www.gpssmartsole.com/

[5] Lee, Y.-D. and Chung, W.-Y. (2009) Wireless Sensor Network Based Wearable Smart Shirt for Ubiquitous Health and Activity Monitoring. Sensors and Actuators B: Chemical, 140, 390-395. http://dx.doi.org/10.1016/j.snb.2009.04.040

[6] Haahr, R.G., Duun, S.B., Toft, M.H., Belhage, B., Larsen, J., Birkelund, K., et al. (2012) An Electronic Patch for Wearable Health Monitoring by Reflectance Pulse Oximetry. IEEE Transactions on Biomedical Circuits and Systems, 6, 45-53. http://dx.doi.org/10.1109/TBCAS.2011.2164247

[7] Reveal LINQ ICM System. http://www.medtronicdiagnostics.com/us/cardiac-monitors/reveal-linq/index.htm

[8] Chan, M., Estève, D. and Campo, E. (2011) Elderly Daily Activity Habits or Lifestyle in Their Natural Environments. Proceedings of the 4 th International Conference on Pervasive Technologies Related to Assistive Environments.

[9] Charlon, Y. (2014) Conception de dispositifs électroniques portés pour le suivi de l'état de santé des personnes âgées. Ph.D. Thesis, Université de Toulouse III.

[10] Hajjine, B., Escriba, C., Campo, E., Zedek, S., Acco, P., Soto Romero, G., Hemeryck, A. and Fourniols, J.-Y. (2015) Development of an Electronic Patch for Falls Detection and Elderly Tracking. International Conference on Biomedical and Health Informatics (ICBHI), 9-10 October 2015, Haikou.

[11] Sigfox. http://www.sigfox.com/fr

[12] Hajjine, B., Escriba, C., Acco, P., Soto Romero, G., Campo, E., Filhol, D., Gaudon, A., Bories, L. and Fourniols, J.-Y. (2016) Integration of a Dual GPS and ISM Antenna PIFA in a 
Human Body Patch for Elderly Tracking. Open Journal of Antennas and Propagation, 4, 34-45. http://dx.doi.org/10.4236/ojapr.2016.42004

[13] ADS. http://www.keysight.com/en/pc1297113/advanceddesign-system-ads?cc=FR\&lc=fre

[14] HFSS. http://www.ansys.com/Products/Electronics/ANSYS-HFSS

[15] Wireless Power Consortium. http://www.wirelesspowerconsortium.com/

[16] HD-4100 Series.

http://www.hdmicrosystems.com/ec/liquid-polyimides-and-pbo-precursors/products/sub-p roducts/hd-4100-series.html

[17] PI-2600 Series.

http://www.hdmicrosystems.com/ec/liquid-polyimides-and-pbo-precursors/products/sub-p roducts/pi-2600-series.html

[18] Hajjine, B., Escriba, C., Charlot, S., Hemeryck, A., Roux, J., Zedek, S.F. and Fourniols, J.-Y. (2016) Development of a Printed Coil for Wirelessly Charging a Tracking Elderly Patch. Wireless Engineering and Technology, 7, 83-95.

\section{Submit or recommend next manuscript to SCIRP and we will provide best service for you:}

Accepting pre-submission inquiries through Email, Facebook, LinkedIn, Twitter, etc. A wide selection of journals (inclusive of 9 subjects, more than 200 journals)

Providing 24-hour high-quality service

User-friendly online submission system

Fair and swift peer-review system

Efficient typesetting and proofreading procedure

Display of the result of downloads and visits, as well as the number of cited articles

Maximum dissemination of your research work

Submit your manuscript at: http://papersubmission.scirp.org/

Or contact etsn@scirp.org 